\title{
POLYMYOSITIS FEBRILE BY TOXOPLASMOSIS IN IMMUNOCOMPETENT SCHOOLCHILD: A CASE REPORT
}

\author{
Author: Eneida Q. Veiga ${ }^{1}$ \\ Co-Authors: Alvaro Veiga ${ }^{1}$, Felipe Moliterno ${ }^{1}$, Nathalia Veiga Moliterno ${ }^{1}$, Aline Nogueira Villela \\ Salgado², Guilherme Costa Lyra Porto², Letícia Miloni², Maria Paula Raymundo Cunha Fontes ${ }^{2}$, \\ Ronaldo Coutinho Rocha ${ }^{3}$, Susie Andries Nogueira1, Solimar Stumpf Cordeiro ${ }^{1}$.
}

\begin{abstract}
Background and aims: Toxoplasmosis has a universal distribution affecting more than a third of the world population. To report that although rare, there is a possibility of a diagnostic association of acquired toxoplasmosis and febrile polymyositis in immunocompetent schoolchildren.
\end{abstract}

Methods: Review of medical records and follow-up for further description.

Description: Schoolgirl, 10 years old, female, immunocompetent, with continuous fever and axial myalgia with rapid progression to generalized, with pain to compression of large muscle groups and active and passive mobilization of limbs, with proximal muscle weakness, preserved tendon reflexes. Initial investigations revealed significant alterations such as LDH: 2708-7693 and CPK: 5407. Serologies for negative arboviruses. Excluded pulmonary and renal involvement. Urine 24h with Ptn / Cr ratio: 0.87, Proteinuria: 407 and Clearence of Creatinine: 108. Echocardiogram, abdominal USG and MRI of the skull and neurovertebral axis without alterations. Weight loss: $5 \mathrm{~kg}$. Research of negative autoimmune causes. Serology Toxoplasmosis IgG reagent, low avidity, corroborating for the diagnosis of Acquired Acute Toxoplasmosis. Initiated Sulfadiazine / Pyrimethamine / Folinic Acid and Methylprednisolone. Progressive improvement of the clinical and laboratory conditions was observed after 5 days. Fundoscopy without determinant changes for suspension of corticotherapy. Confirmatory serology: IgM reagent, IgG reagent, increasing titre, re-confirming treatment maintenance. It evolved with rapid recovery of proximal force, wandering before discharge. Remains asymptomatic, in an outpatient follow-up with a fall in the markers of muscle injury.
Conclusions: Toxoplasmosis acquired in the school age, is a diagnostic hypothesis, although rare, should be investigated in the presence of disabling febrile polymyositis

Keywords: Toxoplasmosis; Atypical Toxoplasmosis; Polymyositis; Infectious polymyositis.

1 WOHLFERT, E.A. et. al.; Brains and Brawn: Toxoplasma Infections of the Central Nervous System and Skeletal Muscle. Trends in Parasitology, vol 1631. 2017. California, USA.

2 CUOMO, G.; et. al.; Severe polymyositis due to Toxoplasma gondi in na adult immunocompetent patient: a case report and review of the literature. Springer-Verlag Berlin Heidlberg, vol 21. p 859-862. 2013.

3 PASPALAKI, P.K. et al.; Case Report: Polymyositis and myocarditis associated with acquired toxoplasmosis and immunocompetent girl. BMC Muscleskeletal disorders, 2001 2:8.

4 LONGO, D.L., et al.; Harrison's principles of internal medicine, 18th edition. The McGraw-Hill Companies, 2011.

${ }^{1}$ Professor of Pediatrics, Faculty of Medicine of Petrópolis, Brazil 2Undergraduate medical student of Faculty of Medicine of Petrópolis, Brazil ${ }^{3}$ Pediatrician of Intensive Care of the Teaching Hospital Alcides Carneiro, Brazil

Email:eneida@fmpfase.edu.br 